Journal of Quantitative Spectroscopy

\& Radiative Transfer 79-80C (2003) 1091-1102
Journal of

Quantitative

Spectroscopy \&

Radiative

Transfer

www.elsevier.com/locate/jqsrt

\title{
Scattering of light from atmospheric ice analogues
}

Zbigniew Ulanowski ${ }^{\mathrm{a}, *}$, Evelyn Hesse ${ }^{\mathrm{a}}$, Paul H. Kaye ${ }^{\mathrm{a}}$, Anthony J. Baran ${ }^{\mathrm{b}}$, Rajam Chandrasekhar ${ }^{\mathrm{a}}$

${ }^{a}$ Department of Physical Sciences, University of Hertfordshire, Hatfield, AL10 9AB, UK

${ }^{\mathrm{b}}$ Met Office, Bracknell, Berkshire, RG12 2SZ, UK

Received 3 June 2002; received in revised form 10 September 2002; accepted 11 September 2002

\section{Abstract}

9 New analogues replacing atmospheric ice crystals in light scattering measurements have been developed. These include thin, hexagonal glass fibres and both simple and complex microcrystals resembling cirrus ice, including columns, plates, rosettes and aggregates with a variety of sizes. Results of angle-dependent scattering measurements on the fibres and on levitated crystals are presented, including phase functions, polarization

13 properties and the asymmetry parameter.

(C) 2002 Published by Elsevier Science Ltd.

15 Keywords: Light scattering; Ice crystals; Analogues; Cirrus; Levitation; Asymmetry parameter

\section{Introduction}

17 The significance of ice and mixed-phase clouds to the earth-atmosphere radiation balance and climate is well established. Yet, present understanding of cirrus with regard to scattering properties

19 of ice crystals is weak, which is mainly due to inadequate theoretical models and lack of knowledge concerning in situ size and shape. The areas of applicability of current models are uncertain with respect to the size parameter and crystal shape. For larger size parameters accurate models either do not exist, have not yet been adequately verified or are computationally very demanding. Therefore, measurements of the scattering properties of ice crystals are of paramount importance if phenomena such as cirrus-climate interaction, including the magnitude and sign of radiative forcing, are to be understood.

Field measurements of scattering on ice in clouds are not only difficult but for correct interpretation they require the knowledge of the size and shape of observed crystals. This information is imprecise, especially for smaller sizes. Laboratory measurements on ice crystals are also very demanding as

\footnotetext{
* Corresponding author. Tel.: +44-1707-284604; fax: +44-1707-284644.

E-mail address: z.ulanowski@herts.ac.uk (Z. Ulanowski).
} 
1 the particles have to be maintained in equilibrium with water vapour at low temperatures. Moreover, control over ice crystal morphology is poor [1,2]. The measurement of scattering on non-spherical

3 particles poses further challenges, e.g. the need to present the particle in a specific orientation or, more appropriately, a set of representative orientations: taking a single "snapshot" is not sufficient.

5 Hence, the particle should be immobilized on the one hand, preferably using a non-contact method, and its alignment should be adjustable on the other hand. Here, a useful technique is electrodynamic

7 levitation, where gravity on particles in air is counteracted by forces produced by a system of electrodes [1-3].

9 We have recently proposed to circumvent the difficulties associated with preparing and handling real ice crystals by the use of materials that are stable at room temperature and crystallize in similar

11 habits to ice. Furthermore, objects of higher refractive index can be used, immersed in such a medium that the relative refractive index becomes similar to that of ice in air, broadening the choice

13 of materials [4]. While machined, low refractive index ice analogues have previously been used in light scattering measurements, the samples produced were macroscopic, and as such could only

15 serve as models of particles in the geometric optics regime [5]. Alternatively, longer wavelengths, for example in the microwave range, can be used to obtain desired values of refractive index,

17 absorption and size parameter [6], but few experimental facilities allowing this type of measurement exist. The ice crystal analogues we have developed are appropriate for use at optical wavelengths.

19 They include microcrystals suitable as a direct replacement of both simple and complex ice crystals, as well as thin, hexagonal glass fibres which can be used with water as an immersion liquid to

21 produce data for the testing of theoretical models [4]. We present here results of angle-dependent light scattering measurements on the fibres and on levitated ice-like crystals, including phase functions

23 and polarization properties.

\section{Results and discussion}

\subsection{Hexagonal fibres}

Long, thin fibres with arbitrary shape of cross-section can be obtained by heating appropriately 27 formed glass rods and drawing them out using apparatus designed for producing glass microtools. We have adapted for this purpose a design due to J.A. Reyniers [7] where a glass sample held vertically

29 is heated by a helical coil and stretched by applying a weight. If the temperature is adjusted so that the glass can be extended but remains highly viscous, the cross-section of the original rod can be 31 preserved. Our samples were prepared by first grinding and polishing optical glass (Schott SF-10, $n=1.746$ at $488 \mathrm{~nm}$ ) into hexagonal rods about $1.5 \mathrm{~mm}$ thick and then drawing them out in up

33 to three stages. In this way fibres with sharp edges and flat faces were produced, down to a few $\mu \mathrm{m}$ in diameter-see Fig. 1. This procedure involves finding a balance between obtaining fibres 35 of uniform thickness, which is possible at higher temperatures, and preserving the shape of fibre cross-section (hence sharp edges), which requires lower temperatures. We have found that meeting these contradictory demands requires considerable amount of experimentation to determine optimum heating power and stretching force, as the final outcome is very sensitive to these parameters.

39 Light scattering measurements were carried out using a fibre-optics diffractometer covering the range of scattering angles between $3^{\circ}$ and $177^{\circ}$ [8]. A multi-line Ar-ion laser was the light source. 


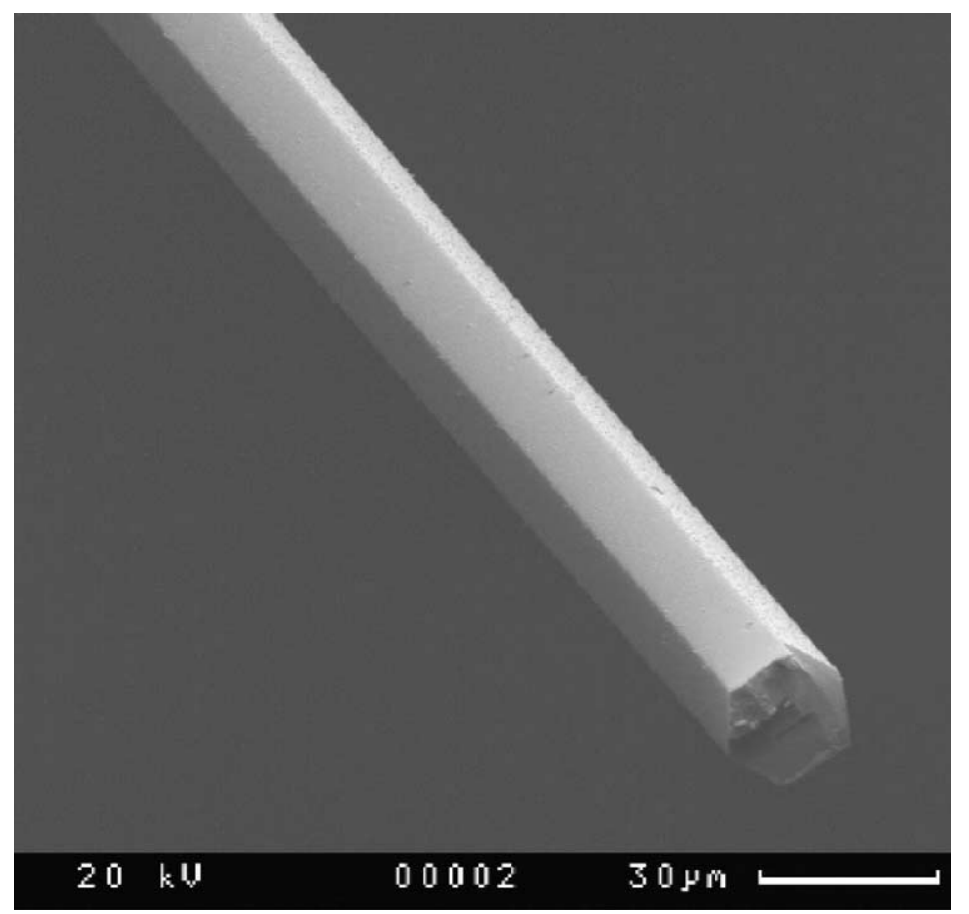

Fig. 1. SEM micrograph showing cleaved end of a hexagonal glass fibre about $25 \mu \mathrm{m}$ in diameter. The visible section is about $500 \mu \mathrm{m}$ long.

1 Dual-wavelength half-wave and quarter-wave plates were used to control the state of polarization, and a prism to select the wavelength, of incident light. The fibres were mounted on a two-axis goniometric cradle attached to a motorized rotation stage. The fibre axis was normal to the scattering plane, except in the special case of tapering fibres - see below. For some experiments the fibres were

5 immersed in water to obtain relative refractive index close to 1.31. Fig. 2 shows a phase function measured in water for a section of the hexagonal fibre from Fig. 1 with a diameter of about $25 \mu \mathrm{m}$,

7 illuminated with a laser beam normal to the fibre axis and with a $8.5^{\circ}$ incidence relative to a facet. The peaks visible near $28^{\circ}$ and $78^{\circ}$ are due to refraction and external reflection, respectively. Given

9 for comparison are phase functions computed using pure geometric optics (GO) ray tracing [9], and a recently developed GO model enhanced by internal and external diffraction [10]. The GO phase

11 function (trace 3 in Fig. 2) illustrates major contributions from refracted and reflected rays which, due to fixed fibre orientation, appear at discrete angles. The enhanced phase function (trace 2) shows

13 how the geometric optics contributions are broadened by diffraction but does not account for some features such as the splitting of the refraction peak near $28^{\circ}$ or the ripple structure between $120^{\circ}$ and

$15160^{\circ}$. These can be interpreted as originating from coherent effects such as interference, which the scattering model used does not include-for further discussion see Hesse et al. [10]. Fig. 3 shows

17 the phase function and the degree of linear polarization for the same fibre in air and at randomized orientation angle about the fibre axis, obtained by averaging over $60^{\circ}$ with a $2^{\circ}$ increment. Clearly visible is a "halo" feature (originating from a single passage through a $60^{\circ}$ prism) which due to the 

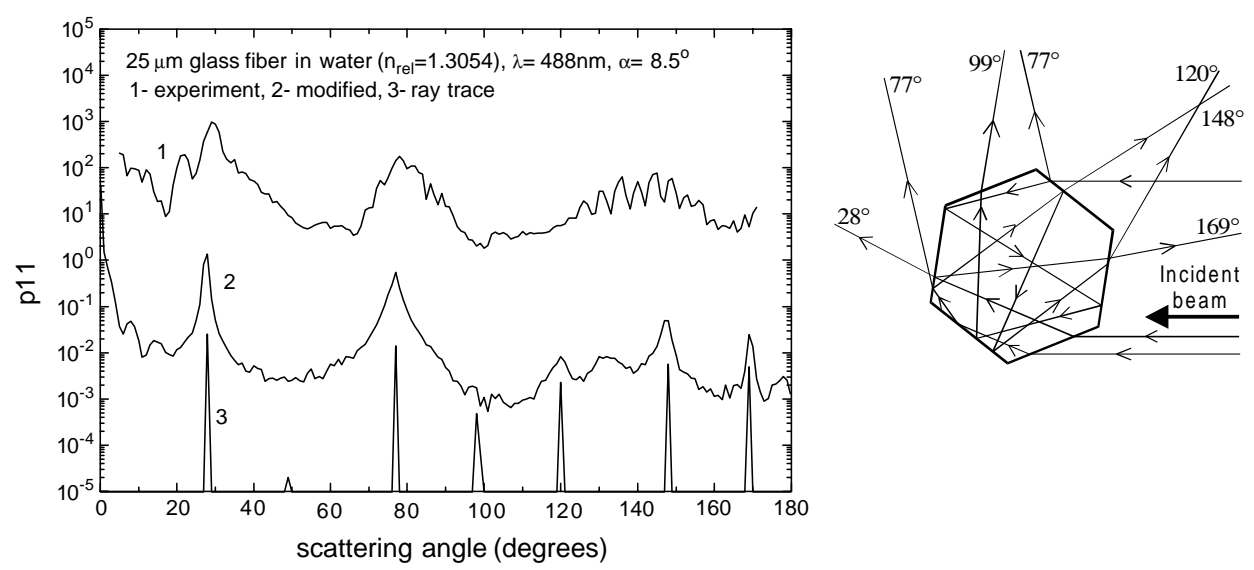

Fig. 2. Measured phase function for a hexagonal fibre at fixed orientation in water (top curve), compared to theoretical models: geometric optics (GO, bottom curve) [9] and GO enhanced by diffraction (middle curve) [10]. The fibre diameter is $25 \mu \mathrm{m}$, incidence normal to fibre axis and at an angle of $8.5^{\circ}$ with respect to a facet, wavelength in vacuum $488 \mathrm{~nm}$ and $n_{\mathrm{r}}=1.305$; the experimental and GO plots are offset in the vertical direction. Shown on the right is the scattering geometry, indicating major ray paths together with corresponding scattering angles.

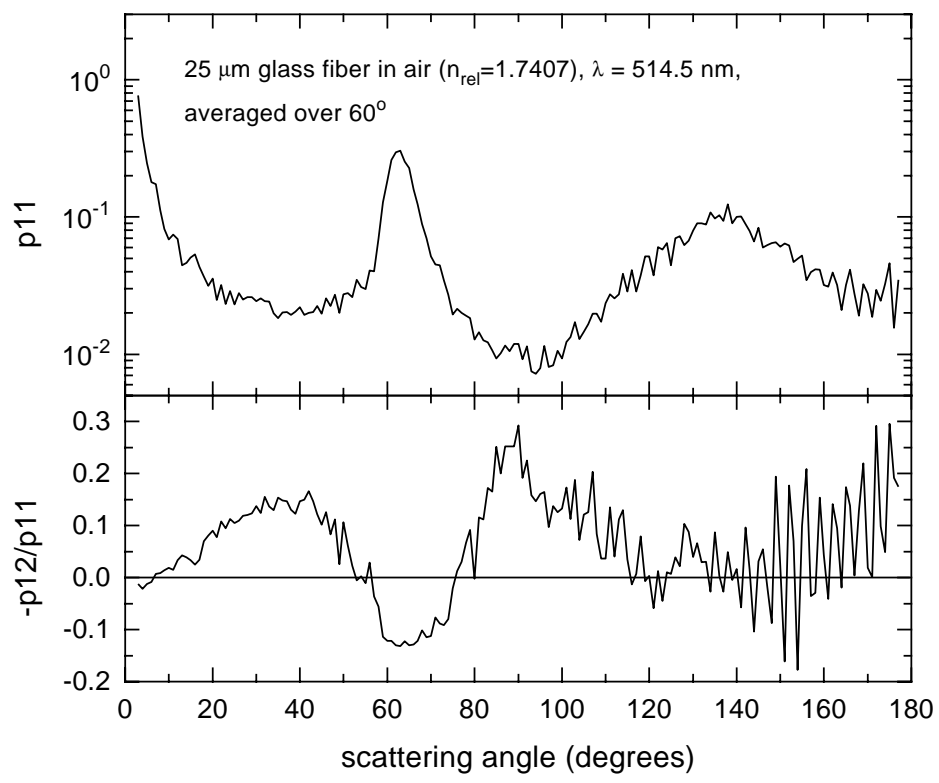

Fig. 3. Measured phase function (upper panel) and degree of linear polarization, defined as the ratio $-p_{12} / p_{11}$, (lower panel) for a hexagonal fibre in air, averaged about the symmetry axis over a $60^{\circ}$ range of angles. The fibre diameter is $25 \mu \mathrm{m}$, incidence normal to fibre axis and the wavelength $488 \mathrm{~nm}$; the phase function has not been normalized.

1 high refractive index in air (1.746) is at an angle of about $61^{\circ}$. Most of the fine ripple at larger scattering angles is an artifact of the averaging, as scattering in that region is dominated by peaks due to external reflections from facets, resulting in a structure with a $4^{\circ}$ period. 


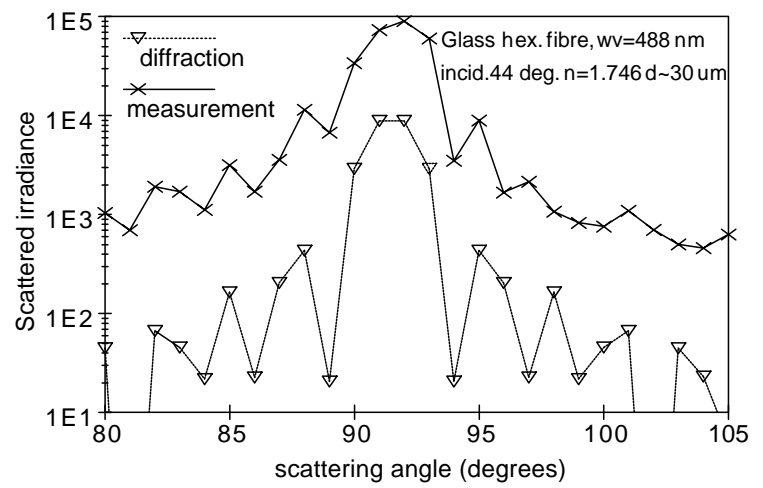

Fig. 4. Scattered irradiance measured for a slightly tapering hexagonal fibre with facets about $15 \mu \mathrm{m}$ wide, illuminated at a wavelength of $488 \mathrm{~nm}$ with incidence direction normal to the fibre axis and at an angle of $44^{\circ}$ with respect to facet normal (top curve). Fraunhofer diffraction on a slit $10.7 \mu \mathrm{m}$ wide representing tilted facet is shown for comparison (lower curve).

While "parallel" fibres of uniform thickness are obviously more useful for testing theoretical models, etc., tapering fibres have some advantages when gaining insight into the mechanism of scattering by faceted objects. This is because multiple internal reflection orders emerge from tapering fibres at different angles with respect to the axis of the fibre and can be visually separated from each other. It is therefore easier to associate particular phase function features with specific families of ray paths. For example, Fig. 4 shows a measured phase function peak due to external reflection on a facet of a fibre with a diameter of about $30 \mu \mathrm{m}$ and tapering at a $1^{\circ}$ angle. The peak has been isolated from internally reflected light by tilting the fibre axis slightly away from the direction normal to the scattering plane. Consequently it is easy to observe the shape of this peak, as determined by wave phenomena and finite width of the facet. This shape can be approximated using Fraunhofer

11 diffraction on a slit $10.7 \mu \mathrm{m}$ wide, representing a facet inclined at $44^{\circ}$ with respect to the incoming beam-see lower curve on Fig. 4. The approximation turns out to be reasonably good, both in terms of peak width and the periodicity of the interference structure, despite the presence of angular aliasing. Observations such as this one have led to the development of a new approximation for

15 scattering on polyhedral objects, based on modifying ray-tracing by the addition of diffraction on facets [10]— see the comparison shown in Fig. 2.

\subsection{Ice-analogue crystals}

From a variety of possible materials fluorosilicates were investigated in greatest depth because their refractive index is close to that of ice at visible wavelengths. Diverse crystal morphologies have been obtained, including hexagonal columns, plates, rosettes and aggregates with a variety of sizes (from less than $1 \mu \mathrm{m}$ to hundreds of $\mu \mathrm{m}$ )-see Figs. 5 and 6 for example scanning electron microscope (SEM) images. The raw material for the crystals shown in Figs. 5 and 6 is sodium hexafluorosilicate $\mathrm{Na}_{2} \mathrm{SiF}_{6}$. It was synthesized by gradually adding $10 \%$ solution of sodium carbonate $\mathrm{Na}_{2} \mathrm{CO}_{3}$ to an aqueous solution containing $17 \%$ fluorosilicic acid $\mathrm{H}_{2} \mathrm{SiF}_{6}$ and $17 \%$ triethanol amine $\left(\mathrm{HOCH}_{2} \mathrm{H}_{2}\right)_{3} \mathrm{~N}$, until a $\mathrm{pH}$ of 3.5 was reached. The precipitate was aged for $2 \mathrm{~h}$ at $100^{\circ} \mathrm{C}$ and either filtered through 

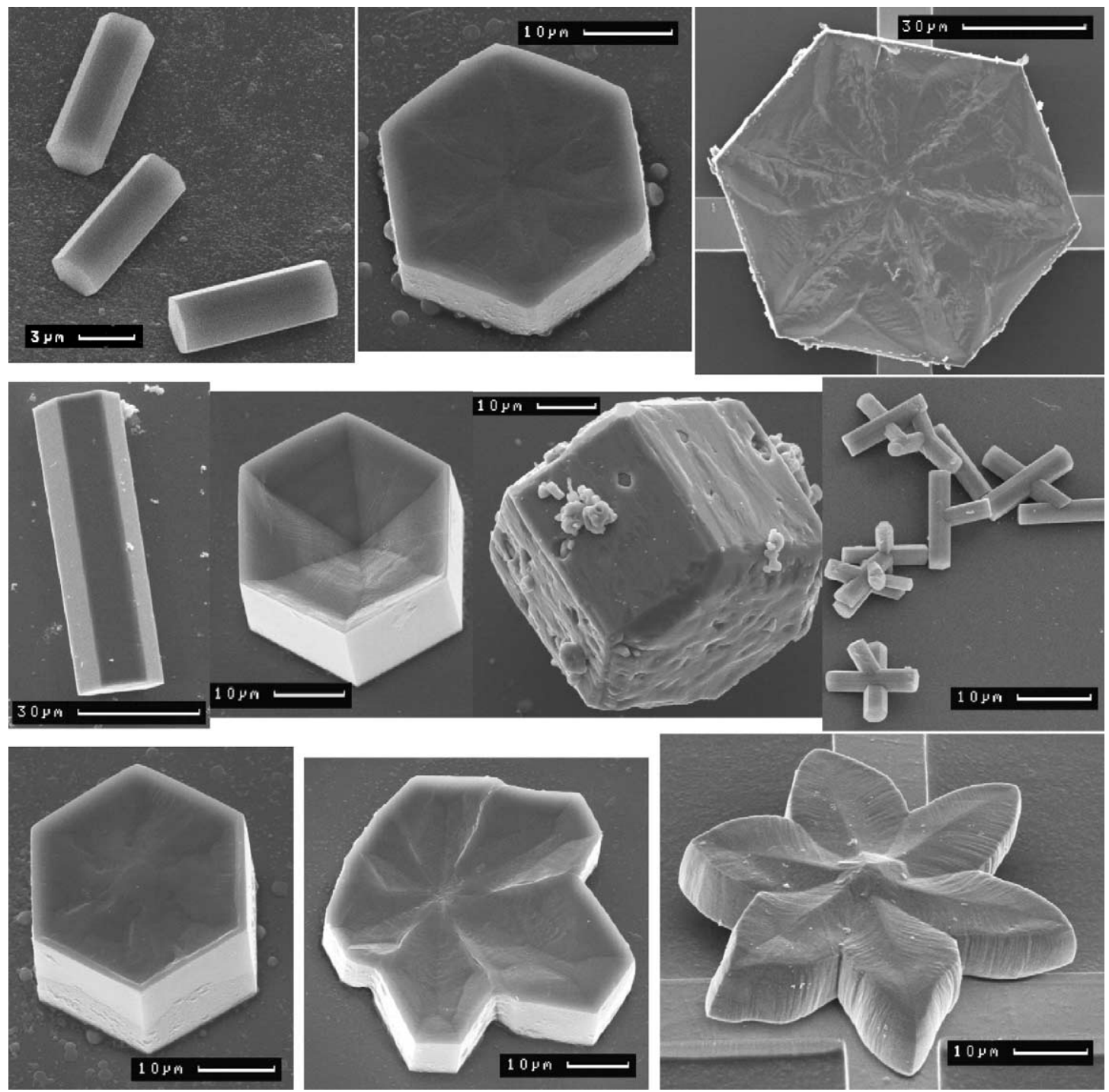

Fig. 5. SEM images of example ice analogues with refractive index of about 1.31 -mainly simple crystals.

1 filter paper or centrifuged. The wet slurry was washed with hot water and centrifuged five times, then dried to a powder at $100^{\circ} \mathrm{C}$. For crystal growth typically $0.2 \mathrm{~g}$ of the powder was shaken in $315 \mathrm{ml}$ of filtered water till partly dissolved and a small amount of the solution was spread over glass microscope slides. The slides were dried at room temperature and a variety crystals were seen to grow. The procedure was varied from the basic one described here to encourage the formation of a wider variety of crystal shapes and sizes.

7 The refractive index of the analogue crystals was measured using immersion refractometry. A phase contrast microscope with blue-green illumination was used for observing crystals immersed

9 successively in four alcohols with refractive index between 1.316 and 1.383 - see Fig. 7. A plot of 

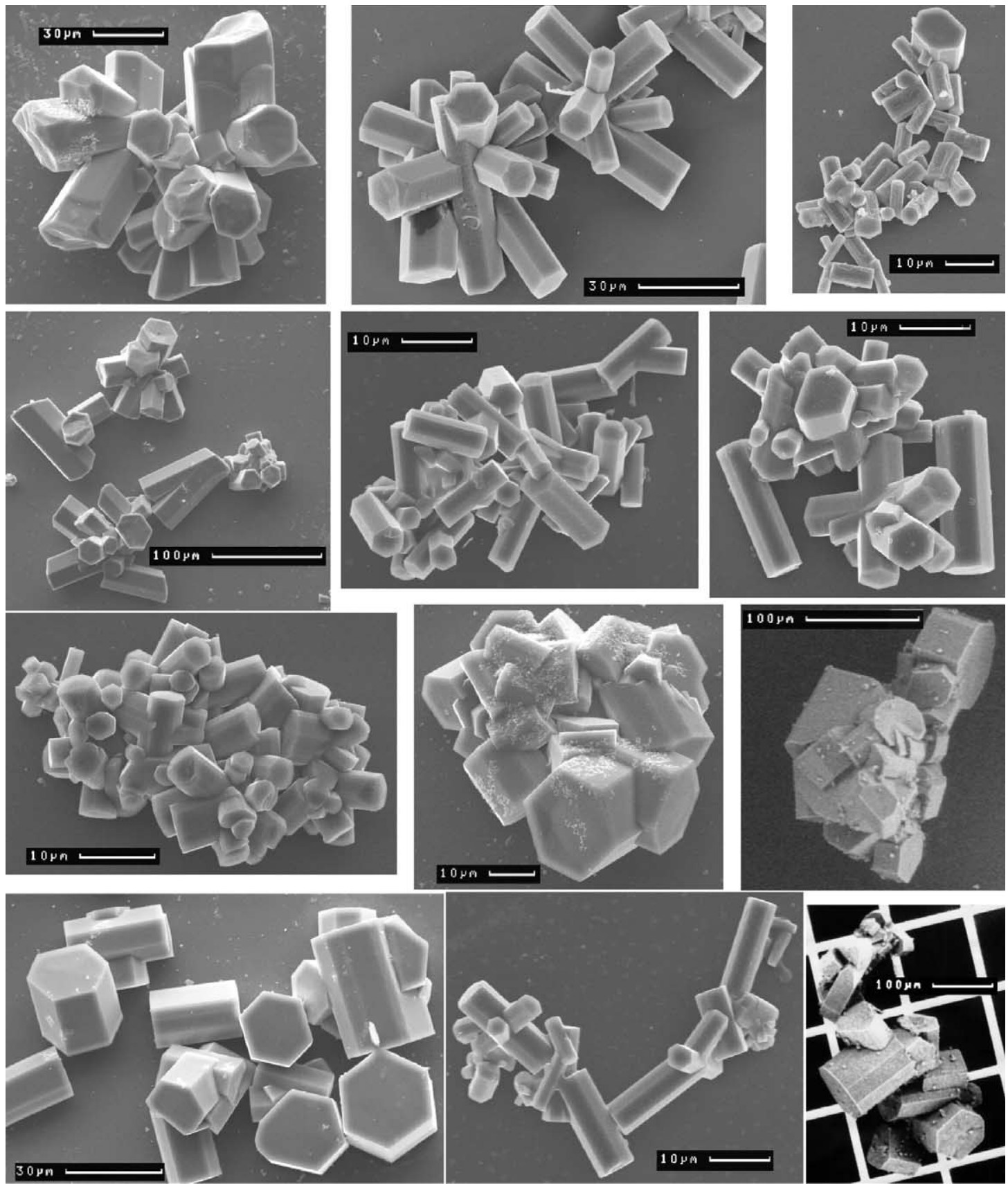

Fig. 6. SEM images of example ice analogues with refractive index of about 1.31 -complex crystals.

1 image brightness measured for two hexagonal columns and a hexagonal plate, the latter about $10 \mu \mathrm{m}$ in diameter and $3 \mu \mathrm{m}$ thick, is shown in Fig. 8. The intercepts with the horizontal axis (where the

3 objects would have the same brightness as the background) indicate the value of refractive index 


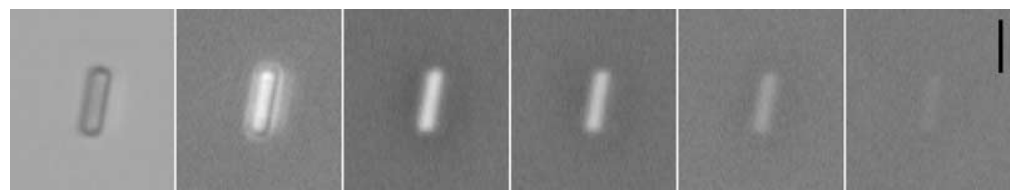

Fig. 7. Optical microscopy images of an ice-analogue column in immersion fluids of decreasing refractive index. The left-hand image is bright field, the remaining ones phase contrast in, left to right: air, isopropanol, ethanol, methanol and octafluoropentanol. The scale bar is $5 \mu \mathrm{m}$.

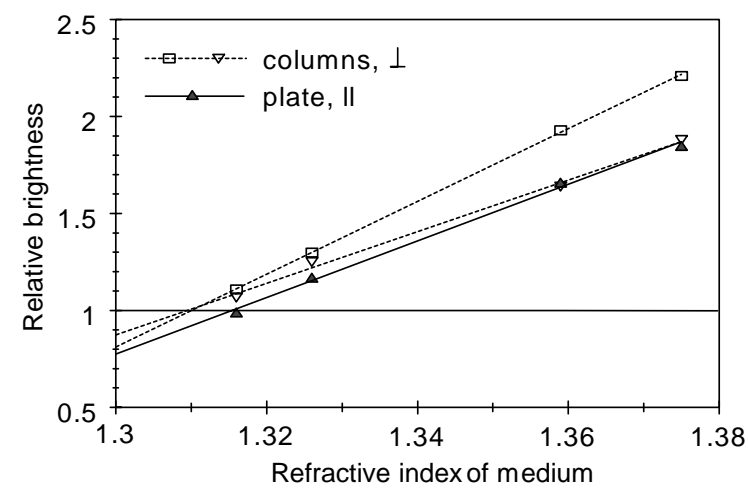

Fig. 8. Brightness of ice-analogue crystal images under phase contrast plotted against refractive index of the immersion medium. Symbols indicate measured values and lines least-square fits.

$1 \quad n$ in the direction perpendicular to the plane of the image. Thus the column has $n=1.310 \pm 0.002$ (perpendicularly to the hexagonal axis) and the plate $n=1.315 \pm 0.002$ (along the axis), pointing to

3 a small amount of negative birefringence in this material. In comparison, ice has a refractive index of 1.313 at $500 \mathrm{~nm}$ and very weak positive birefringence [11].

5 Scattering measurements on the crystalline analogues were carried out using the same setup as for the fibres, except that the crystals were levitated using a specially developed electrodynamic balance

7 (EDB). The experimental procedure is shown schematically in Fig. 9. Single crystals of desired shape and size were selected using micromanipulation with a fine tungsten needle and transferred to

9 copper grids for storage. The crystals were then picked from the grids using a similar needle and injected into the EDB. The EDB allowed presenting the crystals at several orientations. Furthermore,

11 a method for crystal recovery after measurements was developed, so that further examination became possible, e.g. using SEM. Further details of the EDB design and the procedures used are given by

13 Hesse et al. [3].

As an example of experimental results from the analogue crystals, Fig. 10 gives measured phase

15 functions for a simple four-branched rosette and an aggregate, both with a maximum dimension slightly over $30 \mu \mathrm{m}$. The rosette phase function is an average from five spatial (3D) orientations.

17 The functions have been extrapolated to $0^{\circ}$ and $180^{\circ}$ as described below. It is interesting to observe that these irregular crystals have phase functions that are flat and featureless at larger angles, in

19 common with other types of non-spherical particles [12]. A combined phase function, obtained by averaging the data for the rosette and the aggregate, is shown in Fig. 10c in comparison of with the 


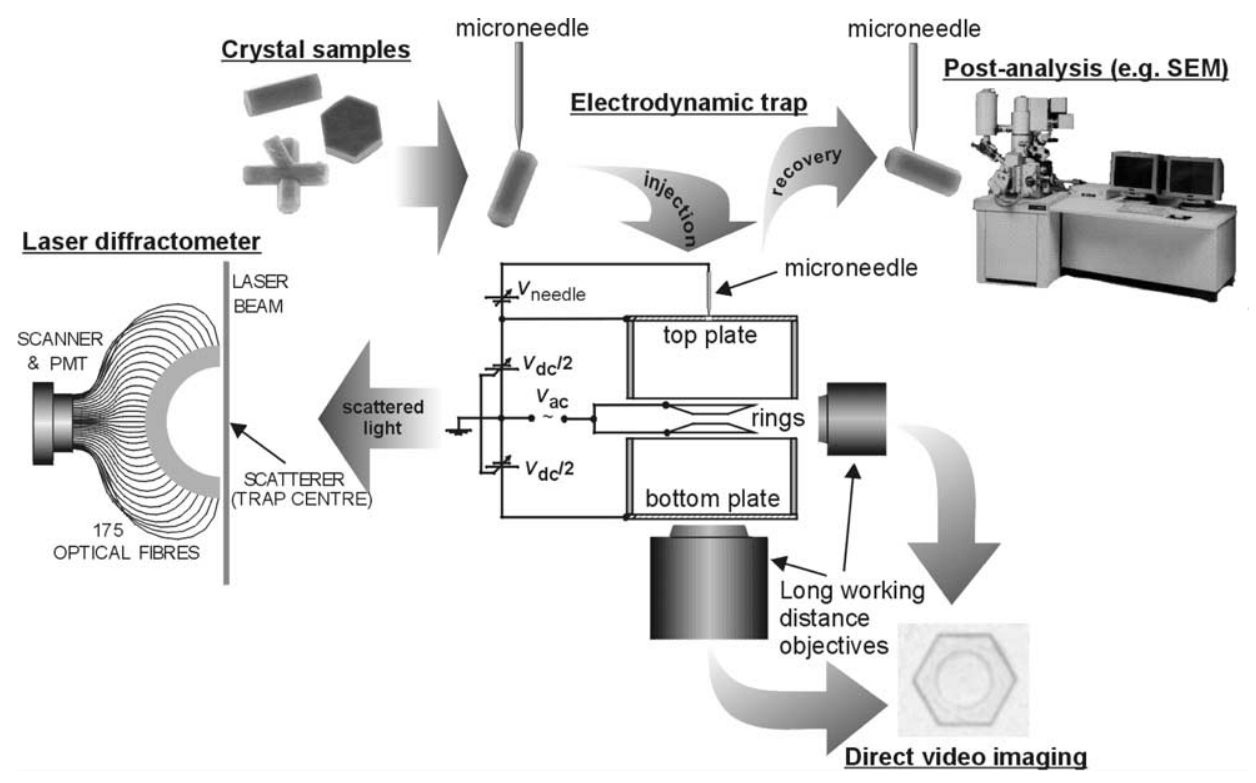

Fig. 9. Experimental procedure used for measurements on levitated ice-analogues crystals.

1 analytic phase function for ice clouds developed by Baran et al. [13]. The parameters of the analytic function have been determined from the asymmetry parameter of the measured function (see below).

3 The analytic and experimental phase functions show good agreement over most scattering angles.

\subsection{Asymmetry parameter}

5 Estimates of the asymmetry parameter $g$ require the knowledge of the full phase function, down to $0^{\circ}$ scattering angle. The present version of the laser diffractometer measures scattered intensities down to a scattering angle of $3^{\circ}$. However, since scattering at such low angles is dominated by diffraction, for randomly oriented particles it is largely independent of shape and approaches like

9 Mie theory can be used to extrapolate the scattered intensity, assuming an equal cross-section sphere [14]. Likewise, Mie theory can approximate the less important $177-180^{\circ}$ region. The cross-section

11 area can be estimated for each crystal orientation from images of the crystal taken before, during or after the measurement. As an example, we have used this procedure to obtain $g$ from experimental

13 data for the smooth rosette in Fig. 10a and found a value of $0.72 \pm 0.03$. This value is consistent with field measurements in an ice cloud consisting of complex bullet rosettes, giving $g \approx 0.74$ [15].

15 Theoretical calculations using the Improved GO method for four-branched bullet rosettes show $g \approx$ 0.79 and similar calculations for smooth rosettes give $g \approx 0.75$ [16], also reasonably consistent

17 with the experimental determination. Using the same procedure as for the rosette, an asymmetry parameter of $0.82 \pm 0.03$ was found for the aggregate phase function from Fig. 10b. Assuming a

19 smooth aggregate of approximately the same projected area the Improved GO method [16] predicts a $g$ value of 0.79 - which is within the lower part of our range of estimated $g$. In contrast, the equal

21 projected area sphere has $g \approx 0.88$. The average of the two measured phase functions shown in Fig. 10c has $g=0.74$. 

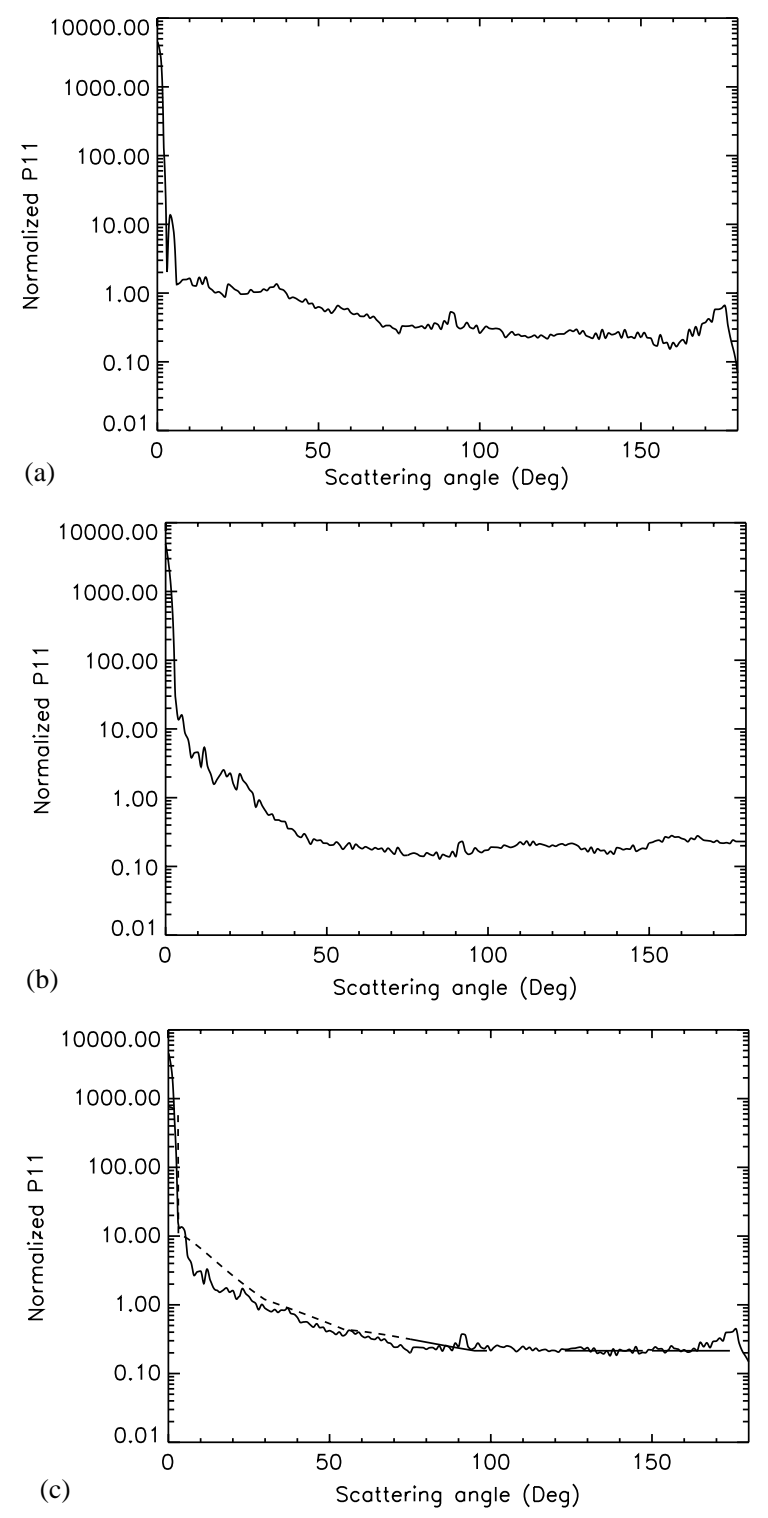

Fig. 10. Measured phase functions for ice-analogue four-branched rosette at randomized orientation (a), simple aggregate (b) and the average of the two phase functions (c), full line. The functions are extrapolated to $0^{\circ}$ and $180^{\circ}$ as described in Section 2. For comparison, the analytic phase function developed by Baran et al. [13] is shown in (c), dashed line. The incident wavelength is $488 \mathrm{~nm}$.

\section{3. Conclusions}

Two new analogues intended for replacing ice crystals in laboratory measurements have been 3 developed. The first one, hexagonal glass fibres, can be used with water immersion as models for long hexagonal ice columns or in air for general study of scattering phenomena and models. The 
1 second one, crystals with refractive index very close to that of ice at visible wavelengths, can be used as a replacement for atmospheric ice crystals in scattering measurements. A variety of crystal shapes typical of cirrus ice have been produced, including pristine shapes, like columns and plates, as well as complex ones, comprising mainly rosettes and aggregates of various form. The crystals can be obtained in a range of sizes. The diversity of the material produced in combination with the ability to select individual crystals for measurements allow great flexibility in the study of light scattering on atmospheric ice, while at the same time maintaining a one-to-one relationship between crystal shape and size and the scattering properties of the crystal. Precise control over the analogues, including size, shape and orientation, permits rigorous testing of scattering models.

Measurements on fixed hexagonal fibres at normal incidence and electrodynamically levitated crys-

11 tals, including complex ones, have been carried out. Long fibre results have been used for the testing of theoretical models. They have also motivated the development of a new approximation for scat-

13 tering on faceted objects [10], example results from which are shown in Fig. 2. The measurements on complex analogue crystals show phase functions which are flat at larger scattering angles, in common with other types of non-spherical particles. There is close resemblance between the measured functions and the analytic phase function for ice clouds [13]. These results indicate that the

17 asymmetry parameter of ice crystals in cirrus clouds is likely to be near 0.74 at non-absorbing wavelengths, keeping in mind the approximations made and experimental uncertainties. The mea-

19 surements confirm that the asymmetry parameter of non-spherical particles is lower than for their spherical counterparts. The ability to measure phase functions down to $3^{\circ}$, in combination with high resolution imaging of analogue crystals, is important in this context because low angle scattering is dominated by diffraction, allowing essentially shape-independent extrapolation of the phase function down to $0^{\circ}$ using estimates of crystal projected area (for randomly oriented particles). Hence the asymmetry parameter can be obtained with reasonably good accuracy.

25 However, it must be stressed that much further work is required to confirm and refine these preliminary conclusions, including measurements on more examples of crystals of various shapes and sizes. To constrain the asymmetry parameter more closely future measurements on the analogues will attempt to reduce the minimum scattering angle to between $1^{\circ}$ and $2^{\circ}$. This will improve the accuracy of the low-angle extrapolation, not least because of lesser dependence on projected area estimates.

\section{Acknowledgements}

We are indebted to Charles Parfitt for producing the fibres, to Mike Cox, Len Banford and Pam Carr for advice on crystal growth, to Andreas Macke for providing the geometric optics program, to John Kerr for assistance with constructing the levitation and scattering apparatus and to Bill Liley for electron microscopy. This research was supported by the Natural Environment Research Council. Anthony Baran was funded by the UK Department of the Environment, Food and Rural Affairs under contract PECD 7/12/37.

\section{References}

[1] Pluchino A. Observations of halo scattering from single ice crystals. Opt Lett 1986;11:276-8. 
1 [2] Bacon NJ, Swanson BD. Laboratory measurements of light scattering by single levitated ice crystals. J Atmos Sci 2000;57:2094-104.

[3] Hesse E, Ulanowski Z, Kaye PH. Stability characteristics of cylindrical fibres in an electrodynamic balance designed for single particle investigation. J Aerosol Sci 2002;33:149-63.

[4] Ulanowski Z, Hesse E, Kaye PH, Baran AJ, Chandrasekhar R, Parfitt C. Scattering of light on ice crystal analogues. In: Proceedings of the Sixth International Congress on Optical Particle Characteristics, Brighton. London: Institute of Physics, 2001. p. 81-2.

[5] Barkey B, Liou KN, Takano Y, Gellerman W, Sokolsky P. An analog light scattering experiment of hexagonal icelike particles. Part II: experimental and theoretical results. J Atmos Sci 1999;56:613-25.

[6] Gustafson BAS. Microwave analog to light scattering measurements. JQSRT 1996;55:663-72.

11 [7] Perfilev BV, Gabe DR. Capillary methods of investigating microorganisms. Edinburgh: Oliver and Boyd, 1969. p. $445-552$.

13 [8] Ulanowski Z, Greenaway RS, Kaye PH, Ludlow IK. Laser diffractometer for single particle scattering measurements. Meas Sci Technol 2002;13:292-6.

15 [9] Macke A, Mueller J, Raschke E. Single scattering properties of atmospheric ice crystals. J Atmos Sci 1996;53: 2813-25.

17 [10] Hesse E, Ulanowski Z. Scattering from long prisms computed using ray tracing combined with diffraction on facets. JQSRT 2003;79-80C:721-32.

19 [11] Hobbs PV. Ice physics. Oxford: Clarendon Press, 1974. p. 202.

[12] Mishchenko MI, Lacis AA, Travis LD. Scattering, absorption, and emission of light by small particles. Cambridge: Cambridge University Press, 2002.

[13] Baran AJ, Francis PN, Labonnote L-C, Doutriaux-Boucher M. A scattering phase function for ice cloud. Q J Royal Met Soc 2001;127:2395-416.

[14] Pollack JB, Cuzzi JN. Scattering by non-spherical particles of size comparable to wavelength: a new semi-empirical theory and its application to tropospheric aerosols. J Atmos Sci 1980;37:868-81.

[15] Gerber H, Takano Y, Garrett TJ, Hobbs PV. Nephelometer measurements of the asymmetry parameter, volume extinction coefficient and backscatter ratio in arctic clouds. J Atmos Sci 2000;57:3021-34.

[16] Yang P, Liou KN, Wyser K, Mitchell D. Parameterization of the scattering and absorption properties of individual ice crystals. J Geophys Res 2000;105:4699-718. 